\title{
Towards a Formal Treatment of Secrecy against Computational Adversaries
}

\author{
Angelo Troina $^{1}$, Alessandro Aldini ${ }^{2}$, and Roberto Gorrieri ${ }^{3}$ \\ 1 Dipartimento di Informatica, University of Pisa, \\ troina@di.unipi.it \\ 2 Istituto STI, University of Urbino, \\ aldini@sti.uniurb.it \\ 3 Dipartimento di Scienze dell'Informazione, University of Bologna, \\ gorrieri@cs.unibo.it
}

\begin{abstract}
Polynomial time adversaries based on a computational view of cryptography have additional capabilities that the classical Dolev-Yao adversary model does not include. To relate these two different models of cryptography, in this paper we enrich a formal model for cryptographic expressions, originally based on the Dolev-Yao assumptions, with computational aspects based on notions of probability and computational power. The obtained result is that if the cryptosystem is robust enough, then the two adversary models turn out to be equivalent. As an application of our approach, we show how to determine a secrecy property against the computational adversary.
\end{abstract}

\section{Introduction}

The recent literature concerning the analysis of security protocols reveals an increasing interest towards the compatibility problem between the computational approach, followed by the cryptography community, and the approach based on the Dolev-Yao model, which is instead followed by the formal methods community (see, e.g., $[2,5,11,22,15,14,18,6])$. In particular, it has been widely recognized that a sort of computational view of cryptography must be introduced in the formal approaches to security analysis based on a purely formal treatment of cryptographic operations. The classical Dolev-Yao model, which is based on the perfect cryptography assumption and the restricted expressive power of the adversary [8], favours a convenient application of formal methods that treat cryptographic operations as purely formal. In this view, an encrypted message, which is a formal expression, can be suitably analyzed through techniques borrowed from the fields of, e.g., modal logic and process algebra $[16,12,10,19,17$, 9]. On the contrary, such a model does not take into account that the adversary has (limited) computational resources which can be exploited to obtain data in a way that cannot be captured by, e.g., standard inference rules. The adversary advantage is instead based on notions of probability and computational power.

On the basis of such considerations, we aim at relaxing the conditions of the Dolev-Yao approach to cryptography. In order to overcome such limitations, we 
take into account the probability for a polynomial time adversary of attacking with success a message encrypted with a secret key. While in a Dolev-Yao setting such a possibility is simply disregarded - a message encrypted with an unknown key is a black box - in a real scenario an adversary with a suitable knowledge may have a good chance of obtaining useful information from a ciphertext that, from a purely formal standpoint, is considered to be a black box. By considering the probability of cryptanalyzing a ciphertext, we compare cryptographic expressions through a suitable notion of indistinguishability, which has been introduced in [21]. Such a notion, which is based on a similarity relation, states whether a polynomial time adversary with a certain initial knowledge has a nonnegligible probability of distinguishing two different cryptographic expressions. As a simple example, expressions $\{M\}_{K}$ and $\{\text { rubbish }\}_{K}$ are almost the same if $K$ is secret and the encryption scheme is ideal according to a computational view of what, in practice, perfect cryptography stands for (see, e.g., $[2,11]$ ).

In this paper, we show that the definition of similarity for cryptographic expressions corresponds to the classical Dolev-Yao based notion of equivalence in the case a suitably defined encryption scheme is used that, intuitively, turns out to be robust against any cryptanalysis attack conducted by a polynomialtime adversary. In practice, the intuition is that if the cryptosystem is robust enough, then a computational adversary with a limited amount of resources has the same expressive power of an adversary that does not use cryptanalysis to obtain data. As an application, we show that our notion of similarity can be used to determine the secrecy degree of a message within an encrypted expression.

The rest of the paper is organized as follows. First, we describe how we extended the Dolev-Yao formal model with probabilistic information used to estimate the probability for a polynomial-time adversary of obtaining meaningful information from a ciphertext (Sect. 2). Then, we show a similarity relation that allows cryptographic expressions to be compared from the viewpoint of a polynomial-time adversary (Sect. 3). Afterwards, we present the main theorem showing that such a similarity relation corresponds to the equivalence relation of the Dolev-Yao model in the case the encryption scheme is robust enough (Sect. 4). As an example, we show an application of such an approach to a secrecy problem in system security analysis (Sect. 5). Finally, we conclude the paper by discussing some related work (Sect. 6) and future work (Sect. 7).

\section{Probabilistic Equivalence}

We base our formal model on the Dolev-Yao encryption model defined by Abadi and Rogaway [2]. In this setting, we formulate an extension of the classical equivalence relation among cryptographic expressions that allows for relating those expressions that yield the same information obtained with the same probability

even through cryptanalysis attempts. Therefore, we abandon the usual DolevYao abstraction and we take into account cryptanalysis attacks. 


\subsection{Background}

As a preliminary to our extension, we now introduce the machinery needed to compare cryptographic expressions. We use String to denote a finite set of plaintext messages, i.e. the set of binary strings of a fixed length (ranged over by $m, n, \ldots$ ), Keys to denote a fixed, non-empty set of key symbols (ranged over by $K, K^{\prime}, K^{\prime \prime}, \ldots$ and $K_{1}, K_{2}, K_{3}, \ldots$ ), such that Keys and String are disjoint, and $\operatorname{Exp}$ to denote the set of expressions defined by the grammar:

\begin{tabular}{|cc|}
\hline$M, N::=$ & expressions \\
$K$ & key (for $K \in \mathbf{K e y s})$ \\
$m$ & string (for $m \in \mathbf{S t r i n g}$ ) \\
$(M, N)$ & pair \\
$\{M\}_{K}$ & encryption (for $K \in$ Keys)
\end{tabular}

Intuitively, $(M, N)$ represents the pairing of $M$ and $N$, and $\{M\}_{K}$ represents the encryption of $M$ under $K$ via a symmetric encryption algorithm. Pairing and encryption can be nested, like, e.g., in $\left(\{(m, K)\}_{K_{1}}, K_{1}\right)$.

The entailment relation $M \mapsto N$ says that $N$ can be derived from $M$. Formally, such a relation is inductively defined as the least relation satisfying the following properties:

$$
\begin{array}{ll}
M \mapsto M & \\
M \mapsto N_{1} \wedge M \mapsto N_{2} & \Rightarrow M \mapsto\left(N_{1}, N_{2}\right) \\
M \mapsto\left(N_{1}, N_{2}\right) & \Rightarrow M \mapsto N_{1} \wedge M \mapsto N_{2} \\
M \mapsto N \wedge M \mapsto K & \Rightarrow M \mapsto\{N\}_{K} \\
M \mapsto\{N\}_{K} \wedge M \mapsto K \Rightarrow M \mapsto N
\end{array}
$$

In essence, $M \mapsto N$ expresses what the adversary obtains from $M$ without any prior knowledge of its content. For instance, $\left(\left\{\left\{K_{1}\right\}_{K_{2}}\right\}_{K_{3}}, K_{3}\right) \mapsto K_{3}$, and $\left(\left\{\left\{K_{1}\right\}_{K_{2}}\right\}_{K_{3}}, K_{3}\right) \mapsto\left\{K_{1}\right\}_{K_{2}}$, but $\left(\left\{\left\{K_{1}\right\}_{K_{2}}\right\}_{K_{3}}, K_{3}\right) \not \not K_{1}$. The entailment relation models the expressive power of the adversary based on the Dolev-Yao model and includes all the operations that such an adversary can execute to construct ciphertexts or extract plaintexts.

Our extension consists in taking into account the possibility for an adversary of obtaining meaningful information from a ciphertext $\{M\}_{K}$ without knowing the key $K$. To this purpose, we introduce the probabilistic pattern $P \cdot p$, which represents an expression $P$ that does not contain undecryptable blocks and is associated with a parameter $p \in] 0,1]$, which models the probability of getting the plaintext contained in $P$. Formally, we define the set $\mathbf{p P a t}$ of probabilistic patterns with the grammar:

\begin{tabular}{|cl|}
\hline$P \cdot p, Q \cdot p::=$ & probabilistic patterns \\
$K \cdot p$ & key (for $K \in$ Keys) \\
$m \cdot p$ & string (for $m \in$ String) \\
$(P \cdot p, Q \cdot p) \cdot p$ & pair \\
$p \in] 0,1]$ & \\
\hline
\end{tabular}


A probabilistic pattern associated to a ciphertext is obtained by substituting every ciphered block with the corresponding expression in clear associated with the probability of obtaining information about it. Given a computational polynomial time adversary $\mathcal{A}$ and an initial knowledge $G$, the probabilistic pattern associated with expression $\{m\}_{K}$ is expressed in terms of the probability of obtaining information about $m$, denoted by $m \cdot p_{\operatorname{dec}}\left(\{m\}_{K}, G\right)$. Function $p_{d e c}\left(\{m\}_{K}, G\right)$ returns the probability of obtaining meaningful information from the ciphertext $\{m\}_{K}$ by exploiting the initial knowledge $G$. More formally, an adversary $\mathcal{A}$ with polynomially timed resources and knowledge $G$ has a probability $\operatorname{Pr}$ at most equal to the value expressed by $p_{d e c}$ of retrieving $m$ from $\{m\}_{K}$ :

$$
\operatorname{Pr}\left[m \leftarrow \mathcal{A}\left(\{m\}_{K}, G\right)\right] \leq p_{\operatorname{dec}}\left(\{m\}_{K}, G\right) \text { for all } \mathcal{A}
$$

Note that the outcome of $p_{d e c}$ is a value strictly greater than 0 , because, even if with small probability, an adversary may randomly guess the key. Besides, the value of $p_{d e c}$ depends on the knowledge $G$ exploited to conduct the cryptanalysis attempt. Intuitively, we could figure out the adversary as an arbitrary (probabilistic) algorithm, executing in polynomial time, that makes computations on ciphered blocks in order to get information about the contained plaintext (see, e.g., [11]). Note that the classical Dolev-Yao adversary obtains $m$ from $\{m\}_{K}$ if and only if $K$ can be derived from $G$ : If $G \mapsto K$, then $p_{\operatorname{dec}}\left(\{m\}_{K}, G\right)=1$. On the other hand, in a computational model assuming ideal encryption [11] or type-0 secure encryption scheme [2], $p_{d e c}$ is a negligible function, as it turns out that the probability of guessing information that cannot be derived through the Dolev-Yao model of cryptography is negligible. In the following we will consider a formal definition of negligible function and we will show that if $p_{d e c}$ is negligible, then it holds that the expressive power of the computational adversary is limited by that of the Dolev-Yao adversary and vice versa.

The outcome of function $p_{d e c}$ represents the starting point for the computation of the probability of cracking a ciphered block. Consider, e.g., expression $\left(\left\{\{m\}_{K_{1}}\right\}_{K_{2}},\left\{\left(K_{1}, K_{2}\right)\right\}_{K}\right)$. What is the probability of getting information about $m$ in the case no prior knowledge is available? An immediate answer could be $p_{\operatorname{dec}}\left(\left\{\{m\}_{K_{1}}\right\}_{K_{2}}\right) \cdot p_{\text {dec }}\left(\{m\}_{K_{1}}\right)^{1}$, that is the probability of sequentially cracking the two keys $K_{2}$ and $K_{1}$. However, we observe that if $K$ is a weak key, then information about $K_{1}$ and $K_{2}$ can be easily derived from $\left\{\left(K_{1}, K_{2}\right)\right\}_{K}$ and, as a consequence, the cryptanalysis of $\left\{\{m\}_{K_{1}}\right\}_{K_{2}}$ may be simplified. Hence, the probability of success may vary according to the strategy adopted by the adversary. By considering the worst case, we always associate to a ciphered block the maximum probability of getting information about it, i.e. we take into account the best cryptanalysis strategy from the adversary standpoint. To this end, we analyze all the possible cryptanalysis paths that the adversary can follow. In the

\footnotetext{
${ }^{1}$ For the sake of simplicity, we omit the knowledge $G$ whenever either $G$ is equal to the empty set or the content of $G$ is clear from the context.
} 
next section, we describe through an illustrative example the structures and the functions used to determine the best cryptanalysis strategy [21].

\subsection{Cryptanalyzing a ciphertext}

The methodology that aims at turning an expression $N$ into a probabilistic pattern $N . p$ consists of four steps. In this section we illustrate each step of the methodology through an illustrative example. We consider the expression $N=\left(\left\{\{m\}_{K_{1}}\right\}_{K_{2}},\left\{\left(K_{1}, K_{2}\right)\right\}_{K}\right)$ and we assume that the initial knowledge $G$ does not allow the adversary to derive any information from $N$ through the entailment relation.

The first step of our methodology consists in computing the keys that can be obtained from the expression, possibly with (without) cryptanalysis attempts. In our example, by hypothesis it is not possible to obtain keys through the entailment relation. In other words, the expression is a black box from the viewpoint of a classical Dolev-Yao adversary. In fact, we have that $K$ can be derived with a probability based on $p_{\text {dec }}\left(\left\{\left(K_{1}, K_{2}\right)\right\}_{K}\right)$. Through such a single cryptanalysis, the adversary obtains $K_{1}$ and $K_{2}$ too. Alternatively, the adversary may try to obtain $K_{2}$ by attacking $\left\{\{m\}_{K_{1}}\right\}_{K_{2}}$ with a success probability equal to $p_{\text {dec }}\left(\left\{\{m\}_{K_{1}}\right\}_{K_{2}}\right)$. Afterwards, $\{m\}_{K_{1}}$ may be cracked in a similar way, and so on. In essence, several different strategies can be adopted to derive the keys contained in $N-K, K_{1}$, and $K_{2}$ - from $N$ itself, and each of such strategies must be evaluated.

Formally, given an expression $M$ and the initial knowledge $G$, we denote by $\operatorname{pKeys}_{M}^{G}$ a set of pairs of the form $T \cdot p$, where $T \subseteq$ Keys is a set of keys syntactically occurring in $M$, and $p \in] 0,1]$ is the probability of retrieving the keys contained in $T$ through a certain strategy. Set $\mathbf{p K e y s} \mathbf{G}_{M}^{G}$ is generated through the following two-phase algorithm:

$$
\begin{aligned}
& \text { pKeys }_{M}^{G}=\left\{\operatorname{initKeys}((M, G))_{\cdot 1}\right\} \\
& \operatorname{addK\operatorname {Keys}}((M, G), 1)
\end{aligned}
$$

In the first phase, $\mathbf{p K e y s}_{M}^{G}$ is initialized with the set of keys that can be derived from $M$ and $G$ without cryptanalysis attempts. Formally, initKeys : Exp $\rightarrow$ $\mathcal{P}$ (Keys) takes an expression $L$ and returns the set of keys recoverable from $L$ through the entailment relation. Hence, initKeys $(L)=\{K \in \mathbf{K e y s} \mid L \mapsto K\}$. Then, in the second phase, the probabilities of retrieving the remaining keys contained in $M$ are calculated. Formally, $\operatorname{addKeys}(H, p)$, with $H \in \operatorname{Exp}$ and $p \in] 0,1]$, is defined through the following algorithm:

$$
\begin{aligned}
& \operatorname{addKeys}(H, p)::= \\
& \forall\{N\}_{K}:\left(H \mapsto\{N\}_{K} \wedge H \not \neg K\right) \text { do begin } \\
& p^{\prime} \quad=p \cdot p_{\operatorname{dec}}\left(\{N\}_{K}, H\right) \\
& L \quad=(H, K) \\
& T \quad=\{K \in \text { Keys } \mid L \mapsto K\} \\
& \operatorname{pKeys}_{M}^{G}=\operatorname{pKeys}_{M}^{G} \cup\left\{T \cdot p^{\prime}\right\}
\end{aligned}
$$

end 
At each step of the algorithm above, a cryptanalysis is performed that reveals, with a certain probability, new keys obtained from $M$. In particular, for each cryptanalysis strategy, $\mathbf{p K e y s} \mathbf{s}_{M}^{G}$ contains the set of keys violated by following that strategy and the probability of cracking such keys.

The second step of our methodology consists in computing the maximum probability of retrieving from an expression a given set of keys by following the best cryptanalysis strategy. In our example, the maximum probability of guessing $K$ is equal to the probability of cracking $\left\{\left(K_{1}, K_{2}\right)\right\}_{K}$, because this is the only strategy that can be followed to obtain $K$. On the other hand, the maximum probability of guessing $K_{2}$ is the maximum between $p_{\operatorname{dec}}\left(\left\{\{m\}_{K_{1}}\right\}_{K_{2}}\right)$ and $p_{\text {dec }}\left(\left\{\left(K_{1}, K_{2}\right)\right\}_{K}\right)$, which are the probabilities associated with the two possible strategies that can be followed to obtain $K_{2}$.

Formally, given an expression $M$, the initial knowledge $G$, and a set $T$ of keys included in $M$, we denote by $\operatorname{Guess}_{M}^{G}(T)$ the maximum probability of cracking all the keys in $T$ according to the best cryptanalysis strategy that can be followed to attack $M$. Denoted $\operatorname{Keys}(M)$ the set of keys occurring in $M$ and given the set $\mathcal{D}_{p \text { Guess }}^{G}=\{T \subseteq$ Keys $\mid T \subseteq \operatorname{Keys}(M)\}$, we define $\left.\left.p \operatorname{Guess}_{M}^{G}: \mathcal{D}_{p \text { Guess }_{M}^{G}} \rightarrow\right] 0,1\right]$ as:

$$
p G u e s s_{M}^{G}(T)=\max \left\{p \mid J_{\cdot p} \in \operatorname{pKeys}_{M}^{G} \wedge T \subseteq J\right\} .
$$

Note that $p G u e s s_{M}^{G}(\emptyset)=1$ since $\operatorname{initKeys}((M, G))_{\cdot 1} \in \operatorname{pKeys}_{M}^{G}$ and $\emptyset \subseteq$ initKeys $((M, G))$.

The third step of our methodology consists in computing the maximum probability of retrieving all the information contained in a ciphertext. In our example, that means we want to evaluate the maximum probability of getting $m, K, K_{1}$, and $K_{2}$. As it is easy to see, such a probability is equal to $p_{d e c}\left(\left\{\left(K_{1}, K_{2}\right)\right\}_{K}\right)$, because through such a single cryptanalysis it is possible to obtain all the keys used within $N$.

Formally, given an expression $M$ and the initial knowledge $G$, we denote by $p M a x_{M}^{G}$ the maximum probability of getting the information contained in $M$ :

$$
\operatorname{pMax}_{M}^{G}=p G u e s s_{M}^{G}(\operatorname{Keys}(M)) .
$$

The fourth step of our methodology consists in turning each ciphered block of an expression into a probabilistic pattern. The obtained probabilistic patterns associate each plaintext with the maximum probability of obtaining it. In our example, the ciphertext $\left\{\left(K_{1}, K_{2}\right)\right\}_{K}$ is turned into the probabilistic pattern $\left(K_{1 \cdot p}, K_{2 \cdot p}\right) \cdot p$ where $p=p G u e s s_{M}^{G}(\{K\})=p_{\text {dec }}\left(\left\{\left(K_{1}, K_{2}\right)\right\}_{K}\right)$ and the ciphertext $\left\{\{m\}_{K_{1}}\right\}_{K_{2}}$ is turned into the probabilistic pattern $m \cdot p$ Guess ${ }_{M}^{G}\left(\left\{K_{1}, K_{2}\right\}\right)$.

Formally, given an expression $M$, the initial knowledge $G$, and an expression $M^{\prime}$ contained in $M$, we denote by $p P_{M}^{G}\left(M^{\prime}, T\right)$ a function that $(i)$ returns in $T$ the set of keys needed to obtain the plaintext contained in $M^{\prime}$ and (ii) associates to such a plaintext the maximum probability of obtaining it through the best cryptanalysis strategy that can be applied to $M$. Function 
$p P_{M}^{G}: \operatorname{Exp} \times \mathcal{D}_{p G u e s s_{M}^{G}} \rightarrow \mathbf{p P a t}$ is defined inductively as follows:

$$
\begin{array}{lll}
p P_{M}^{G}(K, T) & =K_{p \text { Guess }}^{G}(T) & (K \in \text { Keys }) \\
p P_{M}^{G}(m, T) & =m \cdot p \text { Guess }_{M}^{G}(T) & (m \in \text { String }) \\
p P_{M}^{G}\left(\left(N_{1}, N_{2}\right), T\right) & =\left(p P_{M}^{G}\left(N_{1}, T\right), p P_{M}^{G}\left(N_{2}, T\right)\right)_{p \text { Guess }_{M}^{G}(T)} & \\
p P_{M}^{G}\left(\{N\}_{K}, T\right) & =p P_{M}^{G}\left(N, T^{\prime}\right) & \left(T^{\prime}=T \cup\{K\}\right)
\end{array}
$$

Finally, given an expression $M$ and the initial knowledge $G$, the probabilistic pattern associated to $M$ is given by $p P_{M}^{G}(M, \emptyset)$. In the following, we use the abbreviation $p P_{M}^{G}$ (with no arguments) to stand for $p P_{M}^{G}(M, \emptyset)$.

The values $p M a x_{M}^{G}$ and $p P_{M}^{G}$ yield different information that are both meaningful to relate cryptographic expressions. Consider the following example ${ }^{2}$ :

$$
M=\left(\{m\}_{K},\{n\}_{K}\right) \quad N=\left(\{m\}_{K},\{n\}_{K^{\prime}}\right), \quad K \neq K^{\prime} .
$$

which yield the same probabilistic patterns. Indeed:

$$
p P_{M}=\left(m_{\cdot \hat{p}}, n \cdot \hat{p}\right) \cdot 1,
$$

where $\hat{p}=p \operatorname{Guess}_{M}(\{K\})=\max \left\{p_{\operatorname{dec}}\left(\{m\}_{K}\right), p_{\operatorname{dec}}\left(\{n\}_{K}\right)\right\}$. The intuition is that an adversary can crack $M$ by guessing $K$, which is used to cipher both blocks. However, if $\operatorname{pGuess}_{M}(\{K\})=\operatorname{pGuess}_{N}(\{K\})=\operatorname{pGuess}_{N}\left(\left\{K^{\prime}\right\}\right)$ we also have that:

$$
p P_{N}=\left(m_{\cdot \hat{p}}, n \cdot \hat{p}\right) \cdot 1 .
$$

Hence, $M$ and $N$ have the same probabilistic patterns, even if to get in clear the whole expression $N$ an adversary should guess two different keys, namely $K$ and $K^{\prime}$. Such a difference is captured by the fact that:

$$
\operatorname{pMax}_{M}=p \operatorname{puess}_{M}(\{K\})=\hat{p} \neq \hat{p}^{2}=\operatorname{pGuess}_{N}\left(\left\{K, K^{\prime}\right\}\right)=\operatorname{pMax}_{N} .
$$

Therefore, $\operatorname{pMax}_{M}^{G}$ is needed to express the overall probability of getting the whole plaintext, while $p P_{M}^{G}$ is needed to associate each piece of information contained in an expression with the probability of getting it in clear.

In the following, we show how the information computed through the methodology surveyed above can be exploited to compare cryptographic expressions.

\subsection{Equivalence}

Given the expressions $M$ and $N$ and an initial knowledge $G$, we say that $M$ and $N$ are probabilistically equivalent $\left(M \approx^{G} N\right)$ if they yield the same probabilistic pattern and if $p M a x_{M}^{G}$ and $p M a x_{N}^{G}$ are equal.

Definition 1. $M \approx^{G} N \Leftrightarrow p P_{M}^{G}=p P_{N}^{G} \wedge p \operatorname{Max}_{M}^{G}=p M a x_{N}^{G}$.

\footnotetext{
${ }^{2}$ We assume an empty knowledge and omit $G$.
} 
Example 1. Consider the expressions $N=\left(\left\{\{m\}_{K_{1}}\right\}_{K_{2}},\left\{\left(K_{1}, K_{2}\right)\right\}_{K}\right)$ and $M=$ $\left(\{m\}_{K_{1}},\left\{\left(K_{1}, K_{2}\right)\right\}_{K}\right)$, and assume an empty initial knowledge. If $K$ is weaker than $K_{1}$, we have that $p_{\text {dec }}\left(\{m\}_{K_{1}}\right) \leq p_{\text {dec }}\left(\left\{\left(K_{1}, K_{2}\right)\right\}_{K}\right)$ and $p G u e s s_{M}\left(\left\{K_{1}\right\}\right)=$ $p \operatorname{Guess}_{M}(\{K\})=p_{\text {dec }}\left(\left\{\left(K_{1}, K_{2}\right)\right\}_{K}\right)$. Therefore, given $\hat{p}=p_{\text {dec }}\left(\left\{\left(K_{1}, K_{2}\right)\right\}_{K}\right)$, we have $p P_{M}=\left(m_{\cdot \hat{p}},\left(K_{1 \cdot \hat{p}}, K_{2 \cdot \hat{p}}\right) \cdot \hat{p}\right) \cdot{ }_{1}$. On the other hand, from the previous examples and from the condition $p_{\text {dec }}\left(\{m\}_{K_{1}}\right) \leq p_{\operatorname{dec}}\left(\left\{\left(K_{1}, K_{2}\right)\right\}_{K}\right)$, we obtain the probabilistic pattern $p P_{N}=\left(m_{\cdot \hat{p}},\left(K_{1 \cdot \hat{p}}, K_{2 \cdot \hat{p}}\right) \cdot \hat{p}\right) \cdot 1$ and, since $\operatorname{pMax}_{M}=$ $\operatorname{pMax}_{N}=\hat{p}$, we also obtain $M \approx N$. In conclusion, we observe that ciphering the first block $m$ of $N$ with both keys $K_{1}$ and $K_{2}$ is not meaningful, since $N$ is probabilistically equivalent to an expression where this information is ciphered with one of those keys only. Indeed, an adversary can gain information about $m$ by cryptanalyzing the second block $\left\{\left(K_{1}, K_{2}\right)\right\}_{K}$.

\section{Indistinguishability among Cryptographic Expressions}

The notion of equivalence presented above is not adequate to state the indistinguishability among cryptographic expressions. On the one hand, it is not realistic to require that the same ciphered blocks have to be decrypted exactly with the same probability. On the other hand, it is not worth considering those blocks that can be decrypted with negligible probability, since essentially they are almost equivalent to a black box.

In order to relax the notion of equivalence for cryptographic expressions, we introduce a relation, called $\varepsilon$-probabilistic similarity $\left(\approx_{\varepsilon}\right)$, which $(i)$ approximates the equivalence by introducing a tolerance to small differences of the probabilistic parameters that are associated with the probabilistic patterns, and (ii) allows for equating the black box and those ciphertexts that can be decrypted with a negligible probability.

Given an initial knowledge $G$, we say that $M$ and $N$ are $\varepsilon$-probabilistically similar $\left(M \approx_{\varepsilon}^{G} N\right)$ if $p \operatorname{Max}_{M}^{G}$ and $p M a x_{N}^{G}$ are almost the same up to the tolerance $\varepsilon$ and if $M$ and $N$ are $\varepsilon$-compatible according to the notion of compatibility $\sim_{\varepsilon}$ specified below.

Definition 2. $M \approx_{\varepsilon}^{G} N \Leftrightarrow p P_{M}^{G} \sim_{\varepsilon} p P_{N}^{G} \wedge\left|p \operatorname{Max}_{M}^{G}-p M a x_{N}^{G}\right| \leq \varepsilon$.

The compatibility relation $\sim_{\varepsilon}$ for probabilistic patterns expresses when two probabilistic patterns are indistinguishable. Formally, it is defined as follows:

\begin{tabular}{|llc|}
\hline$P_{\cdot p} \sim_{\varepsilon} Q \cdot p^{\prime}$ & if $p, p^{\prime} \leq \varepsilon$ & $P \cdot p, Q \cdot p^{\prime} \in$ pPat \\
$K \cdot p \sim_{\varepsilon} K \cdot p^{\prime}$ & if $\left|p-p^{\prime}\right| \leq \varepsilon$ & $K \in$ Keys \\
$m \cdot p \sim_{\varepsilon} m \cdot p^{\prime}$ & if $\left|p-p^{\prime}\right| \leq \varepsilon$ & $m \in$ String \\
$\left(P \cdot p_{1}, Q \cdot p_{2}\right) \cdot p_{3} \sim_{\varepsilon}\left(P^{\prime} \cdot p_{1}^{\prime}, Q^{\prime} \cdot p_{2}^{\prime}\right) \cdot p_{3}^{\prime}$ & if $\left|p_{3}-p_{3}^{\prime}\right| \leq \varepsilon$ & $\wedge$ \\
& $P \cdot p_{1} \sim_{\varepsilon} P^{\prime} \cdot p_{1}^{\prime} \wedge Q \cdot p_{2} \sim_{\varepsilon} Q^{\prime} \cdot p_{2}^{\prime}$ \\
& $P \cdot p_{1}, Q \cdot p_{2}, P^{\prime} \cdot p_{1}^{\prime}, Q^{\prime} \cdot p_{2}^{\prime} \in \mathbf{p P a t}$
\end{tabular}

Note that two different pieces of information are indistinguishable if they are associated with probabilistic parameters that are smaller than the given tolerance $\varepsilon$, i.e. in practice both of them are considered to be a black box. 
For instance, according to such a notion of probabilistic similarity, the expressions $M=\{m\}_{K}$ and $N=\{n\}_{K^{\prime}}$ are indistinguishable if - given $G=\emptyset$, $p P_{M}=m \cdot p_{1}, p P_{N}=n \cdot p_{2}$, and a fixed threshold $\varepsilon$ - the probabilities $p_{1}$ and $p_{2}$ are equal or smaller than $\varepsilon$. Also the expressions $M=\{m\}_{K}$ and $N=\{m\}_{K^{\prime}}$ are indistinguishable if $p_{1}=p_{\text {dec }}\left(\{m\}_{K}\right)$ and $p_{2}=p_{\text {dec }}\left(\{m\}_{K^{\prime}}\right)$ are similar (even if not exactly the same). In practice, if $\left|p_{1}-p_{2}\right| \leq \varepsilon$, then $M \approx_{\varepsilon}^{G} N$.

Proposition 1. Given $M, N \in \operatorname{Exp}$ it holds that:

$$
M \approx^{G} N \quad \Rightarrow \quad M \approx_{\varepsilon}^{G} N \quad \forall \varepsilon \in[0,1[.
$$

Proof. See [21].

Proposition 2. Given $M, N \in \operatorname{Exp}$ it holds that:

$$
M \approx^{G} N \quad \Leftrightarrow \quad M \approx_{0}^{G} N .
$$

Proof. A trivial consequence of the definition of compatibility relation.

Finally, note that the case $\varepsilon=1$ is not considered, since it is trivial to see that in such a case it follows $\forall M, N \in \operatorname{Exp} M \approx_{1}^{G} N$.

\section{Perfect Cryptography vs. Ideal Encryption}

In this section we show how our notion of similarity is related to a classical DolevYao equivalence relation defined in an environment where perfect cryptography is assumed. In particular, given a notion of ideal encryption, we will show that if two expressions are equivalent within a classical Dolev-Yao model that relies on perfect cryptography, then the two expressions will also be probabilistically similar if we assume ideal encryption.

\subsection{Equivalence within Perfect Cryptography}

In [2], Abadi and Rogaway define an equivalence relation for cryptographic expressions within a formal model where perfect cryptography is assumed. In this section we introduce a formal equivalence for cryptographic expressions which slightly differs from that defined in [2]. The set Pat of patterns is defined as a variant of the set of expressions with the new symbol $\otimes$ (representing a ciphertext that an adversary cannot decrypt) and without the encryption term.

\begin{tabular}{|cc|}
\hline$P, Q::=$ & patterns \\
$K$ & key (for $K \in \mathbf{K e y s})$ \\
$m$ & string (for $m \in$ String) \\
$(P, Q)$ & pair \\
$\otimes$ & undecryptable text \\
\hline
\end{tabular}

Intuitively, a pattern is an expression that does not contain encrypted terms and that may contain some part that an adversary cannot decrypt. Now, we define 
a function $p$ that, given a set of keys $T$ and an expression $M$, computes the pattern that an adversary can obtain from $M$ if the initial knowledge is the set of keys $T$.

\begin{tabular}{lll|}
$p(K, T)$ & $=K$ & (for $K \in$ Keys $)$ \\
$p(m, T)$ & $=m$ & (for $m \in$ String) \\
$p((M, N), T)$ & $=(p(M, T), p(N, T))$ & \\
$p\left(\{M\}_{K}, T\right)$ & $= \begin{cases}p(M, T) & \text { if } K \in T \\
\otimes & \text { otherwise }\end{cases}$
\end{tabular}

Then, given an initial knowledge $G$, we define function $p a t^{G}(M)$, which expresses the pattern obtained from an expression $M$ by exploiting the knowledge $G$, as $\operatorname{pat}^{G}(M)=p(M$, initKeys $((M, G)))$. For example, if $G$ is empty, pat $^{G}\left(\left(\left\{\left\{K_{1}\right\}_{K_{2}}\right\}_{K_{3}}, K_{3}\right)\right)=\left(\otimes, K_{3}\right)$.

Finally, given an initial knowledge $G$, we say that two expressions are equivalent if they yield the same pattern.

Definition 3. $M \cong^{G} N \Leftrightarrow \operatorname{pat}^{G}(M)=p a t^{G}(N)$.

For example, if $G$ is empty, we have $\left(\left\{\left\{K_{1}\right\}_{K_{2}}\right\}_{K_{3}}, K_{3}\right) \cong\left(\left\{\{m\}_{K_{1}}\right\}_{K_{3}}, K_{3}\right)$ since both expressions yield the pattern $\left(\otimes, K_{3}\right)$.

In [20] we defined a probabilistic equivalence relation that is conservative with respect to the one introduced by Abadi and Rogaway. In this paper we decided to define a slight variant of that relation, which we consider more intuitive: Two expressions should be equivalent when they yield the same information, and when such information can be extracted with the same (or similar) probabilities. By taking such a choice we lost the conservativeness with the equivalence of [2]. As we will see in the next section, the notion of probabilistic indistinguishability introduced in this paper is equivalent, under some assumption, to the notion of equivalence defined in this section.

\subsection{Ideal Encryption}

The notion of ideal encryption intuitively assumes that it should be hard for the adversary to decrypt a message ciphered with an unknown key. In other words, the probability of breaking an encrypted message that cannot be derived in the classical Dolev-Yao model should be small. We formalize the concept of small probabilities by introducing the definition of negligible function (see, e.g., [11]).

Definition 4. A function $f: \mathbb{N} \rightarrow \mathbb{R}$ is negligible, if for any polynomial $q$, $\exists \eta_{0} \in \mathbb{N}: f(\eta) \leq \frac{1}{q(\eta)} \quad \forall \eta>\eta_{0}$.

Then, the ideal encryption hypothesis assumes that $p_{\text {dec }}$ must be a negligible function.

Definition 5. An encryption scheme is ideal if and only if

$\forall\{N\}_{K} \in \mathbf{E x p}, \forall G \in \mathbf{E x p}: G \mapsto K, \forall$ polynomial $q: \exists \eta_{0} \in \mathbb{N}$ such that $p_{\text {dec }}\left(\{N\}_{K}, G\right) \leq \frac{1}{q(\eta)} \quad \forall \eta>\eta_{0}$. 
As a consequence, if the assumption of ideal encryption holds, from the definition above we also have that $\forall \mathcal{A}$ :

$$
\operatorname{Pr}\left[N \leftarrow \mathcal{A}\left(\{N\}_{K}, G\right)\right] \leq \frac{1}{q(\eta)} \quad \forall \eta>\eta_{0} .
$$

By following an approach also used in [22], we show that a result holding in the perfect cryptography scenario also holds in our model (and vice versa) if the ideal encryption assumption is taken.

Theorem 1. Given $M, N \in \mathbf{E x p}$, if the assumption of ideal encryption holds for a natural $\eta_{0}$, taken a polynomial $q$ and a natural parameter $\eta>\eta_{0}$, then:

$$
\left.M \cong^{G} N \quad \Leftrightarrow \quad M \approx_{\varepsilon}^{G} N \quad \forall \varepsilon \in\right] \frac{1}{q(\eta)}, 1-\frac{1}{q(\eta)}[.
$$

Proof. $\Rightarrow)$ A proof of the statement could be found in [21].

$\Leftarrow)$ The statement derives by structural induction on the expression $M$ and by observing that, by hypothesis, $M \approx_{\varepsilon}^{G} N \Rightarrow p P_{M}^{G} \sim_{\varepsilon} p P_{N}^{G}$. In the following, we denote by $T_{M}$ the set initKeys $((M, G))$ and by $T_{N}$ the set initKeys $((N, G))$.

$$
\begin{aligned}
& \text { 1. } p P_{M}^{G} \sim_{\varepsilon} p P_{N}^{G}=K_{1} \quad K \in \text { Keys } \\
& \Rightarrow \\
& p\left(M, T_{M}\right)=p\left(N, T_{N}\right)=K \Rightarrow p a t^{G}(M)=p a t^{G}(N) \Rightarrow M \cong{ }^{G} N \\
& \text { 2. } p P_{M}^{G} \sim_{\varepsilon} p P_{N}^{G}=m \cdot 1 \quad m \in \text { String } \\
& \Rightarrow \\
& p\left(M, T_{M}\right)=p\left(N, T_{N}\right)=m \Rightarrow p a t^{G}(M)=p a t^{G}(N) \Rightarrow M \cong G \\
& \text { 3. } p P_{M}^{G}=P \cdot p \sim_{\varepsilon} Q \cdot p^{\prime}=p P_{N}^{G} \quad p, p^{\prime} \leq \varepsilon \quad P_{\cdot p}, Q \cdot p^{\prime} \in \mathbf{p P a t} \\
& \Rightarrow \\
& p\left(M, T_{M}\right)=p\left(N, T_{N}\right)=\otimes \Rightarrow p a t^{G}(M)=p a t^{G}(N) \Rightarrow M \cong{ }^{G} N \\
& \text { 4. } p P_{M}^{G}=\left(p P_{M}^{G}\left(L_{1}, \emptyset\right), p P_{M}^{G}\left(L_{2}, \emptyset\right)\right)_{\cdot 1} \sim_{\varepsilon}\left(p P_{N}^{G}\left(L_{1}^{\prime}, \emptyset\right), p P_{N}^{G}\left(L_{2}^{\prime}, \emptyset\right)\right)_{\cdot 1}=p P_{N}^{G} \Rightarrow \\
& p P_{M}^{G}\left(L_{1}, \emptyset\right) \sim_{\varepsilon} p P_{N}^{G}\left(L_{1}^{\prime}, \emptyset\right) \wedge p P_{M}^{G}\left(L_{2}, \emptyset\right) \sim_{\varepsilon} p P_{N}^{G}\left(L_{2}^{\prime}, \emptyset\right) \\
& \Rightarrow \quad \text { by induction hypothesis } \\
& p\left(L_{1}, T_{M}\right)=p\left(L_{1}^{\prime}, T_{N}\right) \wedge p\left(L_{2}, T_{M}\right)=p\left(L_{2}^{\prime}, T_{N}\right) \Rightarrow \\
& p\left(M, T_{M}\right)=\left(p\left(L_{1}, T_{M}\right), p\left(L_{2}, T_{M}\right)\right)=\left(p\left(L_{1}^{\prime}, T_{N}\right), p\left(L_{2}^{\prime}, T_{N}\right)\right)=p\left(N, T_{N}\right) \Rightarrow \\
& \text { pat }^{G}(M)=\text { pat }^{G}(N) \Rightarrow M \cong{ }^{G} N
\end{aligned}
$$

Under the assumption of ideal encryption, the four cases above include all the interesting situations in which two probabilistic patterns are compatible according to $\sim_{\varepsilon}$. In particular, the condition $\left|p-p^{\prime}\right| \leq \varepsilon$ is always true if $p, p^{\prime} \neq 1$. Indeed, thanks to the ideal encryption assumption stating that $p, p^{\prime} \leq \frac{1}{q(\eta)}$, if $p, p^{\prime} \neq 1$, we have that $p, p^{\prime}<\varepsilon$. Therefore, the three cases $K \cdot p \sim_{\varepsilon} K \cdot p^{\prime}, m_{\cdot p} \sim_{\varepsilon} m_{\cdot p^{\prime}}$ and $\left(P \cdot p_{1}, Q \cdot p_{2}\right) \cdot p \sim_{\varepsilon}\left(P^{\prime} \cdot p_{1}^{\prime}, Q^{\prime} \cdot p_{2}^{\prime}\right) \cdot p^{\prime}$ collapse into the case $P_{\cdot p} \sim_{\varepsilon} Q \cdot p^{\prime}, p, p^{\prime} \leq \varepsilon$ (case 3 of the proof of $\Leftarrow$ )), when $p, p^{\prime} \neq 1$.

Finally, note that we did not consider the cases in which $P \cdot p \sim_{\varepsilon} Q \cdot p^{\prime}$ for some $P, Q \in \mathbf{p P a t}$ with $p=1(p \neq 1)$ and $p^{\prime} \neq 1\left(p^{\prime}=1\right)$. In such cases, the condition $|1-p| \leq \varepsilon\left(\left|1-p^{\prime}\right| \leq \varepsilon\right)$ does not hold, since, by the ideal encryption assumption, $p \leq \frac{1}{q(\eta)}\left(p^{\prime} \leq \frac{1}{q(\eta)}\right)$ and, by the premises of the theorem, $\varepsilon<1-\frac{1}{q(\eta)}$. As a consequence, it is impossible to find a case in which $P \cdot p \sim_{\varepsilon} Q \cdot p^{\prime}$ for some $P, Q \in \mathbf{p P a t}$ with $p=1(p \neq 1)$ and $p^{\prime} \neq 1\left(p^{\prime}=1\right)$. 


\section{Defining Secrecy for Cryptographic Expressions}

In this section we introduce a notion of secrecy of some information within a given expression. Consider for example the expression $M=\left(\{m\}_{K},\{n\}_{K}^{\prime}\right)$. We are interested in evaluating whether the expression $M$ maintains a given secret $m$ assuming that the expression $G$ models the actual knowledge of an adversary ${ }^{3}$. In particular, we are also interested in evaluating the degree of secrecy of $m$ within $M$. Intuitively, we observe that a certain secret $m$ is private in $M$ if the expression $N$, obtained by substituting every occurrence of $m$ in $M$ with $m^{\prime} \neq m$, is similar to $M$. More formally, we provide the following definition.

Definition 6. Given a knowledge modeled by the expression $G$, a certain secret $\alpha$ such that $\alpha \in$ Keys or $\alpha \in \mathbf{S t r i n g}$, a parameter $\varepsilon \in[0,1[$ and an expression $M \in \mathbf{E x p}$ such that $\alpha$ occurs in $M$, we say that $\alpha$ is $\varepsilon_{G}$-secret in $M$ iff the expression $N$ obtained by substituting every occurrence of $\alpha$ in $M$ with the key $K \neq \alpha$ (if $\alpha \in$ Keys) or with the string $m \neq \alpha$ (if $\alpha \in \mathbf{S t r i n g}$ ) is such that $M \approx_{\varepsilon}^{G} N$.

Intuitively, Definition 6, inspired by [1], states that a certain secret $\alpha$ is private within an expression $M$ if an adversary is not able to distinguish $M$ from the expression $N$ obtained by substituting in $M$ every occurrence of $\alpha$ with $\alpha^{\prime} \neq \alpha$. In a sense, that means the adversary is not able to extract $\alpha$ from $M$ with a probability greater than $\varepsilon$. Therefore, if $\alpha$ is $\varepsilon_{G}$-secret in $M$, we can deduce that the adversary with knowledge $G$ can extract $\alpha$ from $M$ with a success probability equal or smaller than $\varepsilon$.

Example 2. Consider the expression $M=\left(\{m\}_{K_{1}},\{K\}_{K_{2}}\right)$ and a knowledge $G=K_{1}$.

On the one hand, we obviously have that $m$ is not $\varepsilon_{G}$-secret in $M$ for any $\varepsilon \in\left[0,1\left[\right.\right.$. Given $N=\left(\left\{m^{\prime}\right\}_{K_{1}},\{K\}_{K_{2}}\right)$ we have that $M \not_{\varepsilon}^{G} N$, in fact $p P_{M}^{G}=$ $\left(m_{\cdot 1}, K_{\cdot p}\right)$ where $p=p_{\text {dec }}\left(\{K\}_{K_{2}},(M, G)\right)=p_{\operatorname{dec}}\left(\{K\}_{K_{2}},(N, G)\right)$ and $p P_{N}^{G}=$ $\left(m^{\prime} \cdot{ }_{1}, K_{\cdot p}\right)$. Since $m \neq m^{\prime}$ we obviously have that $p P_{M}^{G} \chi_{\varepsilon} p P_{N}^{G}$ so that, in practice, $M \not \not_{\varepsilon}^{G} N$.

On the other hand, we have that $K$ is $\varepsilon_{G^{-}}$-secret in $M$ for any $\varepsilon \in[p, 1[$. Given $N=\left(\{m\}_{K_{1}},\left\{K^{\prime}\right\}_{K_{2}}\right)$ we have that $M \approx_{\varepsilon}^{G} N$ for any $\varepsilon \in[p, 1[$, in fact $p P_{M}^{G}=\left(m_{\cdot 1}, K_{\cdot p}\right)$ and $p P_{N}^{G}=\left(m_{\cdot 1}, K^{\prime} \cdot p\right)$. Since $\varepsilon \geq p$ we have that the adversary is not able to distinguish $K$ from $K^{\prime}\left(p P_{M}^{G} \sim_{\varepsilon} p P_{N}^{G}\right)$, so that in practice we have that $M \approx_{\varepsilon}^{G} N$.

\subsection{An Application}

We apply the notion of secrecy within an expression in a very simple real case. Consider a protocol where a server $S$ could be asked to generate a secret key

\footnotetext{
${ }^{3}$ The expression $G$ models, for example, the sequence of messages exchanged within the network until a certain moment, and the set of keys known by the adversary.
} 
and then send it back to the entity $A$ that applied the request. The server also monitors and keeps track of all the messages exchanged in the network.

Assuming that an authentication phase precedes the protocol, we denote with $K_{S A}$ a key shared between the server $S$ and the entity $A$. Finally, we use $t$ to denote a time stamp. The protocol can be described as follows (with the standard notation $A \rightarrow B: M s g$ we denote a message $M s g$ sent by $A$ and received by $B$ ):

$$
\begin{aligned}
& \text { 1. } A \rightarrow S:\{\text { request, } A, S, t\}_{K_{S A}} \\
& \text { 2. } S \rightarrow A:\{K, S, A, t\}_{K_{S A}}
\end{aligned}
$$

where $K$ is the secret key generated by the server.

We now translate the messages exchanged by the protocol into cryptographic expressions, by assuming that $K_{S A}, K \in$ Keys and that request, $A, S, t$ correspond to $r, a, s, t \in \mathbf{S t r i n g}$, respectively. Hence, we have that the protocol exchanges the following expressions:

$$
\begin{aligned}
& \text { 1. }\{(r,((a, s), t))\}_{K_{S A}} \\
& \text { 2. }\{(K,((s, a), t))\}_{K_{S A}}
\end{aligned}
$$

Now, assume that all the messages exchanged in the network are modeled by the formal expression $G$. Then, we apply our notion of secrecy within expressions in order to check whether the expression $\{(K,((s, a), t))\}_{K_{S A}}$ ensures a given degree $\varepsilon$ of secrecy for $K$. To this end, what the server needs to do is to check whether $K$ is $\varepsilon_{G}$-secret in $\{(K,((s, a), t))\}_{K_{S A}}$. The parameter $\varepsilon$ is fixed by the server and represents the security threshold needed to guarantee the secure execution of the protocol. Note that, as the traffic of information within the network increases and the amount of messages ciphered with the shared key $K_{S A}$ gets larger, the server may not guarantee the $\varepsilon_{G}$-secrecy anymore. Our notion of secrecy within expressions is able to capture situations of this kind. Therefore, if at a certain instant of time $K$ is not $\varepsilon_{G}$-secret in $\{(K,((s, e), t))\}_{K_{S A}}$ anymore, the server may, for example, activate a procedure generating a new shared key with the entity $A$ and then send the secret to $A$ encrypted with the fresh key.

\section{Related Work}

The treatment of cryptographic operations within formal models is covered by a quite large body of literature, but most of these efforts do not consider cryptographic operations in an imperfect cryptography scenario.

This work represents a step toward the definition of a formal language with cryptographic primitives and conditional statements for analyzing both unwanted disclosure of data due to the nature of the protocols and information leakage due to the nature of the cryptographic means. In the literature, both probability and computational complexity are studied in formal settings.

Process algebra and computational view of cryptography are combined in [14] where, in the setting of a subset of asynchronous $\pi$-calculus, an asymptotic notion of probabilistic equivalence is defined. The observational equivalence defined 
in terms of such a notion can be related to polynomial time statistical tests, i.e. equivalent processes are indistinguishable from the viewpoint of polynomial time adversaries. Security is then stated in terms of indistinguishability between the protocol under analysis and an idealized protocol specification. More recently, a definition of probabilistic noninterference which includes a computational case has been defined in [5] in the setting of asynchronous probabilistic reactive systems. In particular, computational noninterference means that the advantage of the external observer (which interacts with the system under analysis) for a correct guess of the interfering adversary behavior is a negligible function. A formal notion of computational indistinguishability is also defined in [13] on the basis of a simple model where public outputs are observed in order to infer the content of secret inputs. Finally, [22] compares the classical Dolev-Yao adversary with an enhanced computational adversary which can guess the key for decrypting an intercepted message (albeit only with negligible probability). The two adversaries are shown to be equivalent with respect to a secrecy property. With this work we share the intuition of adding probabilities of breaking a ciphertext within a formal framework. In [22] the authors define a function similar to our $p_{d e c}$ in order to model the probability for a computational adversary of guessing a key. However they abstract away from the particular ciphertext in which the key to be guessed is used as the ciphering key, and they also abstract away from the knowledge the adversary gets. As we do, they also abstract away from how the probability $p_{\text {dec }}\left(\{m\}_{K}\right)$ could be computed.

We finally point out that probabilistic notions of security as well as approximate security properties can be found in the literature (see, e.g., $[10,7,4,3]$ ), but they do not relate probability and cryptographic primitives.

\section{Conclusion}

With this paper we try to put the basis for defining a formal cryptographic language where $(i)$ information leaks due to the weaknesses of the cryptographic primitives can be estimated by employing conditional statements and the equivalence relation presented in Sect. 2, and (ii) probabilistic covert channels can be revealed by verifying noninterference security properties (as done, e.g., in [7, 4]). In particular, the approximate notion of indistinguishability of Sect. 3 can be used (together with an approximate definition of noninterference) to verify whether security properties of cryptographic protocols can be guaranteed at a reasonable degree.

We did not sift through the details of the estimation of the information leakage which is formally expressed by function $p_{d e c}$. We have partially mitigated the effect of such an abstraction by relaxing the probabilistic equivalence through an approximate similarity relation, which allows us to relate expressions that can be considered equivalent up to a given tolerance $\varepsilon$. On the other hand, we have shown that if $p_{d e c}$ is a negligible function, i.e. the encryption scheme is ideal, equivalence in a formal setting assuming perfect cryptography is equivalent to the similarity of our framework. 
Finally, we sketched a method for applying our notion of similarity for the analysis of secrecy of private information within a formal expression. We plan to investigate this approach in a future extension of a formal cryptographic language with our probabilistic similarity relation.

\section{References}

1. M. Abadi, A. D. Gordon, "A Calculus for Cryptographic Protocols: The Spi Calculus", Information and Computation, 148(1):1-70, 1999.

2. M. Abadi, P. Rogaway, "Reconciling Two Views of Cryptography (The Computational Soundness of Formal Encryption)", in Proc. of 1st IFIP Int. Conf. on Theoretical Computer Science, Springer LNCS 1872:3-22, 2000.

3. A. Aldini, M. Bravetti, A. Di Pierro, R. Gorrieri, C. Hankin, H. Wiklicky, "Two Formal Approaches for Approximating Noninterference Properties", Foundations of Security Analysis and Design II, R. Focardi and R. Gorrieri, eds., Springer LNCS 2946:1-43, 2004.

4. A. Aldini, M. Bravetti, R. Gorrieri, "A Process-algebraic Approach for the Analysis of Probabilistic Non-interference", Journal of Computer Security, vol. 12(2), IOS Press, 2004.

5. M. Backes, B. Pfitzmann, "Computational Probabilistic Non-interference", in Proc. of 7th European Symposium on Research in Computer Security, Springer LNCS 2502:1-23, 2002.

6. A. Datta, R. Kusters, J. C. Mitchell, A. Ramanathan, V. Shmatikov, "Unifying Equivalence-Based Definitions of Protocol Security", in Proc. of Workshop on Issues in the Theory of Security, WITS'04, 2004.

7. A. Di Pierro, C. Hankin, H. Wiklicky, "Approximate Non-Interference", in Proc. of 15th Computer Security Foundations Workshop, IEEE CS Press, pp. 1-17, 2002.

8. D. Dolev, A. Yao, "On the Security of Public-key Protocols", IEEE Transactions on Information Theory, 29:198-208, 1983.

9. A. Durante, R. Focardi, R. Gorrieri, "A Compiler for Analysing Cryptographic Protocols Using Non-Interference", ACM Transactions on Software Engineering and Methodology (TOSEM), vol. 9(4):489-530, 2000.

10. J. W. Gray III, "Toward a Mathematical Foundation for Information Flow Security", Journal of Computer Security, 1:255-294, 1992.

11. J. Herzog, "A Computational Interpretation of Dolev-Yao Adversaries", in Proc. of 3rd Int. Workshop on Issues in the Theory of Security (WITS'03), 2003.

12. R. A. Kemmerer, "Analyzing Encryption Protocols using Formal Verification Techniques", IEEE Journal on Selected Areas in Communications, 7(4):448-457, 1989.

13. P. Laud, "Semantics and Program Analysis of Computationally Secure Information Flow", in Proc. of 10th European Symposium on Programming (ESOP'01), Springer LNCS 2028:77-91, 2001.

14. P. Lincoln, J. C. Mitchell, M. Mitchell, A. Scedrov, "A Probabilistic Poly-Time Framework for Protocol Analysis", in Proc. of 5th ACM Conf. on Computer and Communications Security, ACM Press, pp. 112-121, 1998.

15. D. Micciancio, B. Warinschi, "Completeness Theorems for the Abadi-Rogaway Language of Encrypted Expressions", in 2nd ACM SIGPLAN and IFIP WG 1.7 Workshop on Issues in the Theory of Security (WITS'02), Portland (OR), 2002. 
16. J. K. Millen, S. C. Clark, S. B. Freedman, "The Interrogator: Protocol Security Analysis", IEEE Transactions on Software Engineering, SE-13(2):274-288, 1987.

17. L. C. Paulson, "The Inductive Approach to Verifying Cryptographic Protocols", Journal of Computer Security, 6(1-2):85-128, 1998.

18. A. Ramanathan, J. Mitchell, A. Scedrov, V. Teague, "Probabilistic Bisimulation and Equivalence for Security Analysis of Network Protocols", to appear in Foundations of Software Science and Computation Structures (FOSSACS'04), Springer Verlag, 2004.

19. S. Schneider, "Security Properties and CSP", in IEEE Symposium on Security and Privacy, IEEE CS Press, pp. 174-187, 1996.

20. A. Troina, A. Aldini, R. Gorrieri, "A Probabilistic Formulation of Imperfect Cryptography", in Proc. of 1st Int. Workshop on Issues in Security and Petri Nets, WISP'03, 2003.

21. A. Troina, A. Aldini, R. Gorrieri, "Approximating Imperfect Cryptography in a Formal Model", in Proc. of Mefisto Project Final Workshop, Elsevier ENTCS, to appear, available at http://mefisto.web.cs.unibo.it/pubbl.html.

22. R. Zunino, P. Degano, "A Note on the Perfect Encryption Assumption in a Process Calculus", to appear in Foundations of Software Science and Computation Structures (FOSSACS'04), Springer Verlag, 2004. 